\title{
High-tech coal mine canary
}

ne of coal mining's earliest systems for warning of the presence of methane gas, the canary in the coal mine, though low-tech, was extremely effective and rather easy to read: if the bird died, miners had to get out of the shaft. Today, the search for means to monitor for signs of danger continues. At an annual checkup, one could be given a whole battery of tests, each checking for the presence or absence of biological molecules that can be used to warn of potential health threats. These kinds of tests usually need not be done more than once a year, but for some health conditions, such as diabetes, daily monitoring by the patient or his or her caregivers is required. In such cases, sensors need to work rapidly, be easy to use and relatively painless, and provide accurate results. Given the need for such monitoring, sensor technology development has been of keen interest to the medical community for several decades.

For blood-glucose monitoring, recent technologies that show a great deal of promise include the use of electrochemical or optical means to detect glucose. At this stage, however, accuracy remains an issue for both. For optical measures, there are problems with chemicals that have properties similar to those of glucose and thus result in false positive signals. For electrochemical methods, the measurement is based on the electrochemical oxidation of hydrogen peroxide that is produced when glucose is enzymatically broken down. The oxidation or reduction of chemicals other than hydrogen peroxide at the fixed electrode potential of this sensor, however, can generate false readings. A third means for biochemical detection uses a magnetoelastic sensor system whereby a sensor is coated with a $\mathrm{pH}$-responsive polymer, which swells or shrinks depending on the surrounding $\mathrm{pH}$. In response to a magnetic field, this coated sensor produces vibrations at a frequency related to the size of the sensor. Craig Grimes and colleagues at Pennsylvania State University have now applied this technology to detecting glucose levels and have developed a wireless, remote means to assess glucose concentration in solution (1).

Two key features of this sensor are its inexpensive nature and its ease of pro-

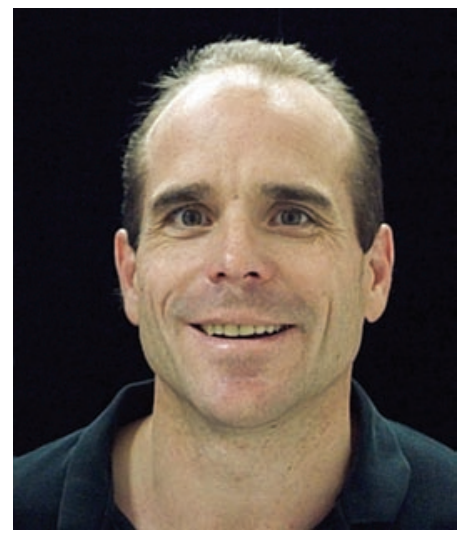

Craig Grimes says that further development in his lab depends on obtaining future funding.

duction. Grimes told the JCI, "The sensor material - you can buy it on a reel, like tape-recording media, and it literally costs about $\$ 250$ a kilometer." The sensor is coated with the $\mathrm{pH}$-sensitive polymer followed by the enzymes or chemicals required to detect the biomolecule of interest. For glucose monitoring, a layer of glucose oxidase is used. Then, in the presence of glucose, glucose oxidation produces gluconic acid, causing the polymer to shrink. The biosensor has a linear response at concentrations between 1-10 $\mathrm{mmol} / \mathrm{l}$ glucose with a detection limit of $0.6 \mathrm{mmol} / 1$.

Another feature of this system - that it is wireless - means that the sensor could be implanted subcutaneously, and glucose levels could be read remotely. This could be done using a detection device no bigger than a wristwatch and would require the capabilities of the sensor reader that Grimes and colleagues have already laid out, although it would have to be reduced to the size of a single chip. "It would cost you about $\$ 12,000$ to do that," Grimes stated. "Then once you have it on a chip, that chip can easily be put into a wristwatch. If you were doing any kinds of economies of scale, it would [cost something like] a Timex Triathlon watch, which is pretty sophisticated and costs maybe $\$ 25$ or so.”

Originally Grimes did not intend to design a glucose sensor; that was something a post-doctoral fellow suggested doing. He initially set out with an eye toward developing hazardous bioagent sensors. "Typically in a bio event," Grimes said, "you have no idea what you are looking for. You don't know what it is, and so most techniques are very specific for one target and money begins to be an issue. So what I had thought was ... you can make an array of five or ten or fifteen of these sensors just as cheaply as one, and the same set of electronics can monitor multiple sensors just as easily as one. George Whitesides at Harvard had developed this really elegant means where you could effectively just print, using an inkjet printer, biorecognition chemistry on surfaces very easily," Grimes added. "So, my vision was [to create] an array of surface-acoustic wave sensors for a dime that you can functionalize... then you can collect a sample, then pop in your sensor [array], and 'boom,' simultaneously test for a lot of different agents, from proteins to pathogens."

In fact, Grimes and colleagues have already begun doing just that, focusing on a list of bioagents that the National Institute of Allergy and Infectious Diseases has indicated it would like the means to detect and quantify. To detect each of these bioagents, the sensor is coated with an immunorecognition and enzyme-amplification system that works along the same principle as that used in a standard ELISA. So far, Grimes and colleagues have been able to detect ricin, Staphylococcus enterococcus $B$, and E. coli 157. "We have gone through very small protein biotoxins up through pathogens using the same sensor platform," Grimes noted. He believes the system could be used for the complete list.

From the perspective of both individual health issues, such as blood-glucose monitoring, and broader population protection from biohazards, the stage of this particular detection platform is set, but for the future, Grimes notes, especially to begin in vivo testing of the glucose biosensor, additional funding is required to move to the next level.

\section{Laurie Goodman}

1. Cai, Q., et al. 2004. A wireless, remote query glucose biosensor based on a $\mathrm{pH}$-sensitive polymer. Anal. Chem. 76:4038-4043. 